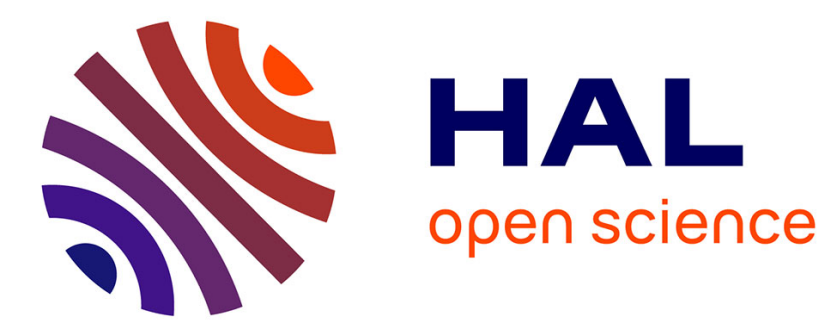

\title{
Prototyping TV and Tablet Facebook Interfaces for Older Adults
}

\author{
José Coelho, Fábio Rito, Nuno Luz, Carlos Duarte
}

\section{To cite this version:}

José Coelho, Fábio Rito, Nuno Luz, Carlos Duarte. Prototyping TV and Tablet Facebook Interfaces for Older Adults. 15th Human-Computer Interaction (INTERACT), Sep 2015, Bamberg, Germany. pp.110-128, 10.1007/978-3-319-22701-6_9 . hal-01599663

\section{HAL Id: hal-01599663 \\ https://hal.inria.fr/hal-01599663}

Submitted on 2 Oct 2017

HAL is a multi-disciplinary open access archive for the deposit and dissemination of scientific research documents, whether they are published or not. The documents may come from teaching and research institutions in France or abroad, or from public or private research centers.
L'archive ouverte pluridisciplinaire HAL, est destinée au dépôt et à la diffusion de documents scientifiques de niveau recherche, publiés ou non, émanant des établissements d'enseignement et de recherche français ou étrangers, des laboratoires publics ou privés.

\section{(c)(1)}

Distributed under a Creative Commons Attribution| 4.0 International License 


\title{
Prototyping TV and Tablet Facebook Interfaces for Older Adults
}

\author{
José Coelho, Fábio Rito, Nuno Luz and Carlos Duarte \\ LaSIGE, University of Lisbon, Portugal \\ \{jcoelho, faarito, nmluz\}@lasige.di.fc.ul.pt, caduarte@fc.ul.pt
}

\begin{abstract}
With the daily problem of social isolation comes an aggravation of older adults' general health. Social Network Services like Facebook have the potential to ameliorate the social connectivity of this segment of the population. However, they are still not fully adopted by them, whether because of agerelated limitations or the lack of appropriate technological skills. In this paper we argue that the development of SNSs based on technology already used by older adults, like Television, or technology which has proven to be more accessible to them, like Tablets, can improve the ability of older adults to use these systems. We report findings from a study composed of semi-structured interviews and focus groups which aimed at the development of two Facebookbased prototypes for TV and Tablet. Results show good receptiveness from older adults to perform social tasks on TV and Tablet-based applications, for interacting using alternative modalities like speech, or back-of-device tapping, and for the use of adaptation mechanisms. Informed by the study results the main contributions are the two prototypes, and a collection of recommendations regarding the design of TV and tablet based interfaces for this population.
\end{abstract}

Keywords. Facebook; older adults; social isolation; tablet; television; semistructured interviews; focus groups; participatory design; prototyping

\section{Introduction}

Older adults face daily a serious problem called loneliness. They spend a lot of time alone in their homes which contributes to increase social isolation [10]. The resulting decrease of social skills can lead to illness or other problems which can increase their mortality risk [2]. Technology can have an important role to improve social abilities in seniors [2]. However, they are quite resistant to adopt new technologies, which hinders the introduction of solutions benefiting social life. Additionally, age-related impairments limit the way these users interact with technology [23]. Online social network services (SNS) have the potential to ameliorate the social life of older adults, allowing users to create and share media content among each other. The main reason for seniors to adopt SNSs is the ability to improve online and offline relationships between them and family and close friends. Facebook, as the most popular SNS [1], constitutes the ideal vehicle for this use. However, a considerable number of older adults still do not adopt or make a limited use of this SNS. Main limitations 
are design complexity $[9,15,20]$, privacy issues $[9,15,16,24]$ and preconceptions about its use $[9,15,20]$ and the inability to use and learn technology used for SNS access [23].

To improve the ability of older adults to access SNSs we argue that its development should be based on technology already used by older adults (like Television) and technology which has proven to be more accessible to them (like Tablets). Television (TV) is the most used technology by older adults [3]. By exploring this close relationship, TV can be used to introduce social applications to older adults. Tablets support direct object manipulation, which has proven to be useful for older adults, because they allow the user to focus only on the screen without requiring attention to the input hardware [28]. Additionally, by being lightweight, small-scaled, and providing internet access everywhere, tablets could help captivate older users [13, 23]. Furthermore, to deal with seniors' disabilities and differences when interacting with technology, we advocate that these social applications should resort to multimodal and adaptive interfaces. Taking the Tablet and TV roles into consideration, as well as the role of multimodality and adaptation, we performed a user study composed by semi-structured interviews and focus group sessions. Main goals were to collect user requirements for a more inclusive design regarding the senior population and to gather information required for the design of two Facebook based prototypes: one TVbased and one Tablet-based. We also wanted to understand preferred alternatives to interact with TV and tablet applications and to evaluate how this population perceives personalization and adaptation concepts.

This paper main contributions are a study which provides relevant indications about how TV and Tablet devices can be used to simplify SNS access among older adults; and two Facebook-based high-fidelity prototypes which aim to ease this access. Concerning the study, results show older adults like to interact with TV and would like to perform social tasks through TV-based applications. When considering Tablet devices, results also indicate that older users would like to interact using several modalities like speech, and back-of-device tapping. The first because it simplifies the interaction without requiring too much effort, while the latter could be especially useful for interacting with the device without hiding information on the screen. Concerning the prototypes, a collection of recommendations is provided regarding the design of TV and tablet based interfaces for this population.

We start by presenting related work on the use of SNS by older adults, and what advantages Tablet and TV can bring to the elderly. We also summarize past research on what multimodal and adaptive interfaces can bring to the older population. Next, we describe the methodology we followed for our study, and follow that with a detailed analysis of the results and respective recommendations for the development of the two prototypes and describe them in detail. We end the paper with the main conclusions. 


\section{Related Work}

\subsection{Older Adults and Social Network Services}

Technology could be an important collaborator to scale down loneliness and consequently to mitigate social isolation. SNS are the most important technology to confront this problem $[2,20]$. There are two distinct types of SNSs that could make older adults more active in social aspects. The first ones are SNSs uniquely designed for older adults however these tend to expire shortly after being launched [15]. The second type are typical SNSs which could be used by older adults if they are designed having this population in consideration like Facebook. Research showed that the first approach excluded older adults' family and close friends which were the main reason for them to adopt SNS in the first place $[15,16]$. Therefore, recently several authors developed solutions based on existing SNSs which can be adapted to the older segment of the population: Tlatoque [9] was an aSNS (ambient SNS), that collected Facebook content and presented it to older adults. It gave support for interacting with SNS using different technologies: a digital frame and a multi-touch PC. Results showed an increase in online and offline relationships between older adults and family; Gomes G. et al. [16] developed a tablet Facebook interface that allowed older adults to see, create and share content with close relatives. They compared it with the Facebook native application for tablets, and showed that older adult's acceptance increased because of the focus on family and close friends. Users also esteemed that content could be filtered by family, close friends and personal content. Privacy and usability issues were also identified as problematic; Norval et al. [24] also explored how SNSs could be accessible for older adults. They presented a comparison between a Facebook application with less functionalities and a SNS designed based on gathered requirements. Their results indicated that the second approach allowed applications to achieve a higher usability level. Users could also perform tasks with less effort and in a shorter time which could lead to an increase of users in SNSs that follow these recommendations. Our prototypes take advantage of the requirements found in these studies that could lead seniors to adopt and use SNSs. Additionally, we will include a new concept of TV shared content that will allow users to share what they are seeing on TV. None of the aforementioned studies attempted to evaluate this context of interaction.

\subsection{Television, Multimodality and Older Adults}

Older adults are often in front of TV and this can lead them to adopt new technologies based on this device $[3,20]$. However, it is crucial to recognize that when interacting with SmartTVs they face problems raised by age-related disabilities [21], Electronic Program Guides (EPGs) [11], or insufficient number of accessible user interfaces [8]. In recent years, several researchers have turned their attention to the potential of TV-based applications: Karahasanovic et al. [19] showed that TV with additional features could be a valid solution for older adults to create and share contents "because it takes less time to turn it on and it should be much simpler to work on"; 
Gaver et al. developed Photostroller [14], an application based on a device that simulated a TV and offered a slideshow of photos retrieved from Flickr. Their findings demonstrated that the use of a remote controller (RC) to interact with the system caused a greater acceptance and that the use of the system increased the number of offline interactions; GUIDE [7] developed a framework to create TV based applications. They showed that multimodality and adaptation could make them more accessible to older adults with disabilities by providing different ways of interaction that fit their characteristics. Their findings also suggested that although RC should be the default way to interact with TV, voice and gestures were seen as better ways to do it; Bobeth J. et al. [3] compared four different techniques for gesture interaction in TV applications. They demonstrated that not only gestures are an accessible modality for older adults to interact with TV applications, but also that older adults prefer an indirect pointing approach to direct pointing, directional gestures or to TV parallel circular movements; finally, the same authors [4], compared the usage of tablet, gestures and RC by older adults in a TV context. They used two applications to show that the use of a tablet to interact with TV applications was appreciated by both older adults and younger people. Gestures were also enjoyed but for very limited interaction, while the RC was appreciated for linear tasks. From all this past research, it is evident the fulcral value of multimodality when concerning more accessible TV-based applications. The You, me \& TV TV-based prototype proposed in this paper builds from this necessity and focuses its design on providing alternative ways of interacting with the TV: RC interaction, speech and gestures.

\subsection{Tablet devices, Multimodality and Older Adults}

Older adults have difficulties using particular devices. They do not have too much strength for holding heavy devices as laptops and the usual computer graphical user interface can also be complicated for them. This is mainly because these interfaces require splitting users' attention between input hardware and the screen content [6]. The use of Tablet devices, by being "eyes-free, button-free, and silent" has been seen as a way to facilitate interaction and increase older adults' accessibility [28]. Other work also showed that Tablet devices offer flexibility which makes adaptation to older adults' characteristics, preferences and heterogeneity easier [11]. Because of these characteristics, the use of Tablet devices as a new bridge between older adults and their social communities has been very successful in recent years. Several projects were based on supporting the exchange of lightweight information, and took advantage of Tablet device features like Internet connectivity, in built-camera and virtual keyboard for capturing, sending and receiving messages and photos for and from older adults' relatives. Examples are applications like PersonCard [21], Wayve [22] and Enmesh [29] or systems like Building Bridges [13]. Other example of the potential of Tablet devices for accessing features typically present in a SNS was Cornejo et al. aSNS [9], which was built on top of Facebook and was also based on a multi-touch screen resembling a tablet. This last example, not only was an incentive for online social relationships but also led to an increase of in-person encounters. Additionally, the majority of these projects was also based on the use of several mo- 
dalities for facilitating interaction with the Tablet. PersonCard and Wayve made use of SMS features, a virtual keyboard and the possibility of performing handwritten messages; Building Bridges focused on adding a Tablet touch, look and feel interface to phone functionalities which made use of speech; even Cornejo's SNS was based on a PC resembling a Tablet and made use of touch interaction, gesture interaction and ambient aware interaction. Like past research focused on TV-based applications for older adults, work performed on Tablets also supports itself on the value of multimodal interaction. Therefore, the Tablet-based prototype proposed in this paper is built with support for a large set of interaction possibilities: touch, gestures, speech, tilting and back-touch interaction. While the first three were already heavily used on past research, the latest two have not been tested on seniors before.

\subsection{Adaptation and Older Adults}

In a similar fashion to multimodal interfaces, adaptation and personalization mechanisms can be used to help older adults interact. From the end of the 20th century, several projects focused on the adaptation and personalization of user interfaces having older adults in mind. This was done either by adapting dynamic characteristics [27], by changing layout and font size [12], or by offering multimodal interaction along with the transformation of applications appearance and presentations style to fit user's characteristics [8]. Moreover, and since about 2009, researchers have been verifying that personalization and adaptation are even more highly appreciated by older adults when considering SNS profile presentation [20] and SNS features [5, 22]. Additionally, other studies focused on the differences concerning awareness and communication needs [19], and regarding distinct chunks of information [17] among older adults. In common, both these studies evidenced the need for these differences to be considered when designing a service usable by older adults, and again, the importance of adaptation mechanisms for this end. Both You, me \& TV and YouTablet prototypes will build from this evidence on the necessity of turning to adaptation features when designing SNS applications more accessible to older adults. Therefore the study reported in this paper will also be concerned with understanding the perception of older adults regarding adaptation mechanisms and chances for personalization.

\section{$3 \quad$ Methodology}

The main goal behind this work is designing two Facebook based prototypes that allow to close the gap between SNSs and older adults. To promote their adoption prototypes will be based on two elderly friendly technologies - Tablet and TV. Both prototypes should be based on design recommendations for seniors collected through previous studies and, given the novel platforms, a further study of our own. This study will heavily involve end-user representatives following a participatory design methodology. This paper covers work developed on the first two phases, the population characterization and the participatory design phases. The last phase involving longitudinal evaluation is under way but will not be focused on this paper. 


\subsection{Population characterization}

To understand which requirements exist for older adults to make use of SNS technology it is paramount to characterize them concerning their technological expertise and age related limitations. We also attend to their opinions on the use of different technologies to access SNS. Consequently, we defined a set of research questions. The first will be concerned about what distinguishes older adults who use Facebook from older adults who do not and what are the main reasons for non-adoption or limited use (RQ1). Other research questions will be concerned with TV and Tablet as vehicles for older adults to adopt SNSs like Facebook. Are the elderly receptive to use social applications on TV (RQ2) or Tablet devices (RQ3)? We are also concerned with what modalities older adults prefer for interacting with TV (RQ4) and tablets (RQ5). Finally, the last research questions will be concerned with the concepts of adaptation and personalization. Which of these concepts is preferred by seniors (RQ6)? What issues should be taken into account in the implementation of each of these concepts (RQ7)? In order to answer the research questions we decided to conduct semi-structured interviews which allowed us to learn more about the population that we are studying. This method allow us to collect more data than simple questionnaires, because when necessary, reasons for a particular response can be asked by the interview conductor.

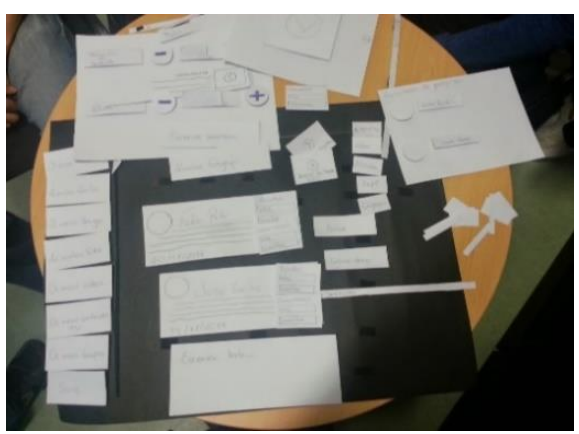

Fig. 1. Low-fidelity prototype of TV inter-

\subsection{Participatory Design}

Once the research questions are answered, it is crucial to gather design recommendations. Those allow us to develop Facebook based prototypes and should be clustered into two distinct groups. A group for the recommendations that improve the development of TV interfaces and other group for the development of tablet prototypes. For that end we decided to carry out focus groups in order to bring older adults to participate in the design process of these prototypes. These focus groups also allow to validate the findings of the interviews before turning them into recommendations to follow for the design of the interfaces. For this validation we developed low fidelity prototypes. The modularity of these prototypes allowed seniors to build together the system's information architecture. Building was achieved by combining elements of the interface represented in pieces of paper over a previously constructed layout (Figure 1). 


\subsection{Validation}

Both prototypes should be validated through tests with older users. The main goal of these tests is to validate if both approaches taken have a social impact in older adults life. Thus, we planned longitudinal tests at older adults' homes to realize their initiative for using the system in real contexts. To measure social isolation we will consider the basis of Cornwell et al. study [10] and their measures of social disconnectedness and perceived isolation. These tests are under way and will run for two to three months.

\section{User Study}

\subsection{Semi-structured Interviews}

Participants. A total of 31 participants (11 male, 20 female) participated in the semi structured interviews. These had a duration of approximately one hour each. All participants were volunteers and more than 60 years of age. Age was not asked directly, participants indicated which of the age ranges they were. There were 6 different categories (Between 60 and 64 (19.35\%), Between 65 and 70 (23.6\%), Between 71 and $75(35.5 \%)$, Between 76 and $80(19.4 \%)$ and More than $80(3.2 \%)$ ). About $93 \%$ of the participants indicated having visual impairments, $36 \%$ have hearing disabilities and about $33 \%$ complained that they forget events that happened some time ago. In a Likert scale of 1 (nothing satisfied) to 5 (very satisfied) participants averaged 3.55 $(\mathrm{SD}=1.31, \mathrm{MED}=4.00$, mode $=5)$ in terms of social satisfaction. Concerning the use of technology, participants reported difficulties, with only $23 \%$ stating to be comfortable using some technology and about $52 \%$ having some to a lot of difficulties interacting with technology. Anonymity was kept at all times, with each user being identified exclusively by an id number. All data was saved in a secure repository.

Procedure. We first inquired older adults about five main fields. First, profiling questions concerning gender, age, education and household composition, selfcharacterization in terms of general and emotional health, social feelings and agerelated impairments were asked. Second, they were inquired about their interest concerning distinct types of technology and about PC, TV and Tablet devices in terms of how easy they are to use in general and in specific tasks. Third, questions related with TV were asked focusing on viewing frequency, TV features, TV-related tasks, and TV content. Older adults were also asked to classify both traditional and alternative ways of interacting with TV. Fifth, participants classified in terms of easiness, satisfaction and effort the different ways of interacting with a Tablet device and ranked their interests in performing typical technological tasks using speech, gesture, or Tablet-base modalities like frame-based interaction, back-touch and tilting. Finally, participants answered to questions related with adaptation and personalization of user interfaces. On a last note, although specific questionnaires for accessing technological expertise and other skills' characterizing instruments already exist we decided against using such instruments due to 1) the increase it would represent in what contributes 
already for a long interview; 2) most of them being based on self-reported data nonetheless.

\subsection{Focus Groups}

Participants. A total of 17 participants ( 3 male and 14 female) participated in the focus groups. The first two focus groups focused on the TV-based prototype, and were composed by two groups of 6 participants. The third focus group focused on the Tablet-based prototype and was attended by 5 participants. Each focus group had a duration of about one hour. All participants were volunteers and more than 60 years of age. From the first two sessions, only one stating to have difficulties interacting with the TV in his daily life. No one saw TV as very easy to interact with. Only 4 in 12 used a PC, with only two considering it easy to use, while two used and considered easy to use the Tablet, and 2 from the 3 who used Facebook considered it easy to use. Thus few participants made use of technologies other than the TV which made them ideal to generate discussion around this platform. On the last focus group, no one had or made use of a Tablet, all classified TV as being easy to use. Three made use of a PC but only one classified it as easy to use, and 3 made use of Facebook, with 2 classifying it as easy to use. This shows this group was more technologically proficient than the other two, however no one had experience with the device on which the discussion was focused.

Procedure. Focus groups were performed at two distinct senior universities and allowed us to define how would the information architecture be organized on both the TV-based (the first two focus groups) and the Tablet-based (the third focus group) Facebook prototypes. Participants were asked to have an active role in changing each detail of the user interface by moving, placing and ordering the distinct components on the cardboard (which simulated the screen). We started by explaining the system and what we wanted to achieve with the discussions. We also asked them to complete a brief questionnaire concerning what technology they use and are familiar with. This served as a basis for characterizing each group. In the first two focus groups, the discussion began around functionalities they see as more important, and how they would like to see the menu and contact information presented. How to group information and contacts and how to filter media content were discussed next. Finally, some discussion was generated around the concepts of personalization and adaptation. In the last focus group, discussion begun with similar topics, the feed functionality, the menu composition and group visualization. More discussion focused around how to perform different tasks in several distinct modalities like speech, back-touch, tilting and touch. Finally, a concept called "digital frame", in which users can watch a slideshow of their contact photos and status updates, was also appreciated by the participants. 


\section{$5 \quad$ User Study Findings}

Results from the structured interviews are presented below. Special consideration is given towards answering each research question identified in the methodology.

\subsection{What distinguishes older adults who use Facebook from those who do not (RQ1)?}

From the thirty elderly individuals interviewed, about half uses Facebook (48.4\%). This serves as an appropriate basis for a comparison between the two populations. We first observed that there are no major differences between them in terms of age-related limitations. Next, we found differences related to the age range of users and nonusers. An association shows that the younger elderly population is the one that uses Facebook more (graphic A on Figure 2). This association is particularly visible from 60 to 71 years of age, as percentage of Facebook users' drops. Further, there is also an association between the use of Facebook and the way participants appreciate using a computer (graphic B on Figure 2), meaning that non users like PC less than Facebook users. In the same way, non-users also like TV more than Facebook users (graphic $\mathrm{C}$ on Figure 2), and they would enjoy less accessing it on a TV context than Facebook users (graphic D on Figure 2) (answers on both, were obtained through Likert scale from 1 (do not like it at all) to 5 (like it very much)). This can be partially explained if we consider that during the interviews it was observed a tendency of non-users to reject all alternatives for accessing Facebook. The main reasons for this population group to not use this SNS are related with privacy issues ("I think that Facebook is not safe. How do I know if talking to a person on Facebook, that person is the only one that sees what I write"). Another reason appointed is related to negative feelings about what people do in the SNS ("I do not like Facebook, because people expose their lives there"). These results showed that younger elderly use Facebook more. Users of this SNS prefer using the computer more than non-users, and vice-versa concerning use of TV. We also found that the reason for non-users of Facebook to reject all alternatives for accessing this SNS, are related with privacy issues and negative feelings about the way that people expose their lives. These findings support previous findings $[9,15,24]$ regarding the use of Facebook by this population.

\subsection{Are the elderly receptive to use social applications on TV (RQ2)?}

The findings about the usage of Facebook, related with the usage of computer and the feelings towards TV already reported when answering RQ1, (chart B and C on Figure 2 respectively) also allow us to discuss that TV could be a way for non-Facebook users to adopt SNS. By not using a computer, they currently cannot make use of Facebook, but their general appreciation towards TV could make this context ideal for the use of SNS. However, when considering their perceived interest in doing so, they rejected it (chart D on Figure 2). Still, as we have seen when answering RQ1, this rejection could be strongly associated with bad feelings about the word "Facebook". In this sense, SmartTV technology could be one way for older adults to access SNS 
like Facebook. However, this technology has not yet reached the older population: 94\% have never used SmartTVs and $71 \%$ do not know what that concept is. Still, when we explained what it is about, they were very enthusiastic. Some of them even said they would buy a SmartTV if they had the chance. From the several tasks they would like to perform using one, tasks related with SNS such as viewing photos and videos of family and close friends, creating events with these groups and sending messages were highlighted. Furthermore, they were also interested in talking to family and friends through video conference, as this would allow them to see people on a larger screen. One participant even said "So I could talk and see my granddaughter on TV when she went to Covilhã". These results are an important outcome of this study as they clearly show that seniors want to perform social tasks through TV. Older adults enjoyed the idea of recording and sharing TV content with family and friends if those contents are related with news, documentaries and debates (Figure 3 (left)). In fact, some of them already do this without this technology: "I'm watching TV and call friends to tell them to switch to what I am seeing". This led to including TV content sharing functionality within the TV based SNS prototype. This also provides excellent feedback to answer this research question, because through a TV-based SNS, older adults have the opportunity to directly share TV contents with their family and close friends. Finally, some older adults proposed a new functionality. They have plenty of printed photos they would like to share with their family and friends through TV. Thus, we have included in the TV-based prototype the possibility to digitize their digital photos and share them through their online network. These results clearly show older adults' interest towards using a TV-based SNS application. Older adults want to make social tasks on TV, such as, talk with their family, sharing TV content and printed photos with their family and close friends.
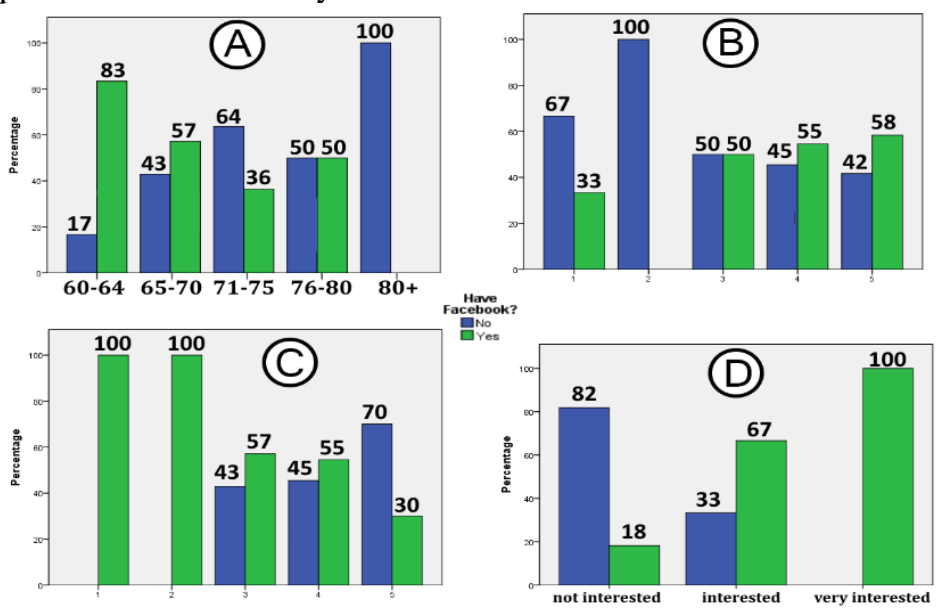

Fig. 2. Differences between users of Facebook and non-users of this SNS: (A) Age distribution; (B) Like using computer; (C) Feelings about TV; (D) Interest accessing SNS in TV. 


\subsection{Are the elderly receptive to use social applications on Tablet devices (RQ3)?}

The majority (77\%) of the participants interviewed said they had never used a tablet but would appreciate the opportunity to do it. In a Likert scale of 1 (I do not like to use a Tablet at all) to 5 (I really like to use a Tablet) they averaged $3.29(\mathrm{SD}=1.54$, $\mathrm{MED}=4.00$, mode $=5$ ). In the same way, the ones who use a Tablet, are very satisfied with the way it works $(\mathrm{M}=4.14, \mathrm{SD}=0.69, \mathrm{MED}=4.00$, mode=4). When questioned about the reasons for using it, they appointed portability associated with talking with family and friends and playing games with their grandchildren. The last two are tasks supported by Facebook. Results from the structured questions regarding easiness of tasks indirectly show that they use it primarily to access the Internet, view and write emails and view photos and videos of family and close friends (Figure 3 (right)). This last functionality is again the main reason appointed by older adults to make use of a SNS like Facebook. However, these results also show that about $71 \%$ of them do not use it to access Facebook. Similar to what happened before regarding the possible use of SNS on TV, this could also have been influenced by the negative feelings towards the word "Facebook". Additionally, negative feelings were also felt concerning privacy issues when using Facebook on a tablet device because of the device specific properties ("I think Facebook in the tablet device is less secure" or "I do not know who sees what I put on Facebook through the tablet device"). Inspired by previous work which made use of Tablet devices as a digital photo frame [9] or as a device for sending and receiving photos from family members [21] we inquired participants about the idea of having it as a photo frame that displays content shared by their family on Facebook. In a Likert scale from 1 (not interesting at all) to 5 (very interesting), they generally enjoyed it, averaging $3.65(\mathrm{SD}=1.52, \mathrm{MED}=4.00$, mode $=5)$. Although results do not clearly show older adults interest in accessing Facebook through a Tablet-based application, the tasks they perform or would like to perform through this device, along with the preconceived negative feelings about Facebook, could indicate the opposite.
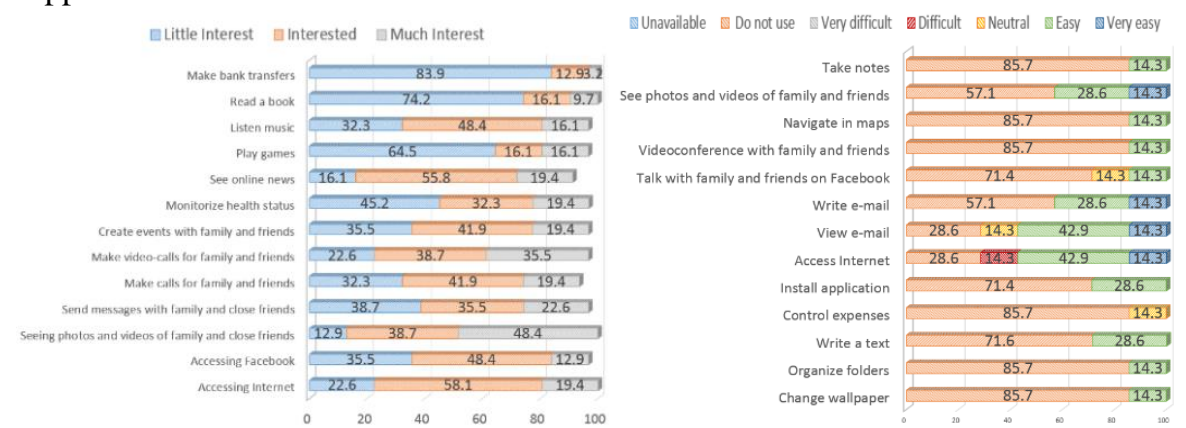

Fig. 3. Interests on sharing TV content (left). Preferred tablet activities (right). 


\subsection{What modalities older adults prefer for interacting with TV (RQ4)?}

When asked about the need for help when interacting with TV-based SNS, older adults showed interest in a broad range of alternatives. The preferred manner of assistance is voice feedback (81\%), followed by help through a virtual character (avatar) (71\%) and textual help (55\%). The preference for voice support was justified as being the one more close to the traditional ways of communication they use daily. Some seniors also underlined that having an application talking to them could make them feel less alone. Several structured questions showed the preferences concerning seniors' interaction with a TV-based SNS. Figure 4 (left) shows older adults prefer both the traditional way (RC) and speech for this interaction but are also open to interact through gestures. Based on these results, we decided to include these two modalities in the TV-based prototype. Finally, in respect to new ways of interaction with the TV, it is important to highlight that seniors found interest to have a tablet as a device for help in two distinct situations (answers were obtained through Likert scale from 1 (totally disagree) to 5 (totally agree)): when they cannot see specific TV-content they would like to have a tablet displaying it at a larger size $(\mathrm{M}=3.81, \mathrm{MED}=4$, mode $=5)$; and for showing content related with what they are watching, meaning providing additional information about TV-content. $(M=4.00, M E D=4$, mode $=4)$. These results show that older adults are strongly interested in having alternative ways of interacting with a TV-based SNS, such as voice and gestures, at the same time as they preserve the traditional interaction (RC) and are helped by contextual information in both au-

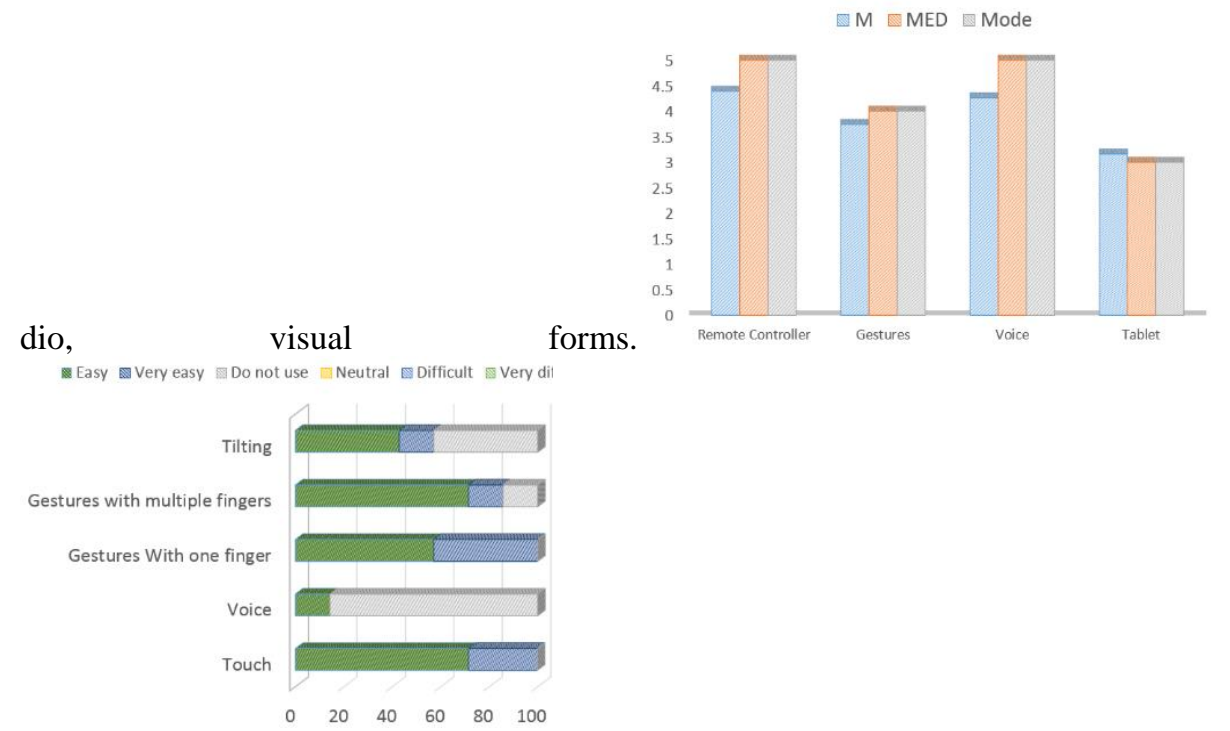

Fig. 4. Older adults preferred modalities for interact with TV (left chart) and tablet (right chart). 


\subsection{What modalities older adults prefer for interacting with Tablets (RQ5)?}

Similar to what happens on a TV context, when asked about the need for help interacting with Tablet-based SNS, older adults showed interest in a broad range of alternatives. Ratios and reasons for preferring speech are the same as for TV interaction, and ratios for avatar and text are also similar. With regard to what modalities older adults prefer to interact with SNS for tablet devices (Figure 4 (right)), seniors appointed first the simplicity of traditional touch interaction as well as gesture interaction using both one or multiple fingers. However, the study obtained some interesting findings that open the possibility to interact in new ways which satisfy the needs of the elderly. About $50 \%$ see tilt interaction as easy or very easy. When asked for further explanation about this, participants told they were used to this way of interaction from when they play games on the tablet device. As a result, we considered providing navigation on the tablet prototype menu by tilting the device. Additionally, voice interaction on the Tablet is almost unknown to older adults. However, when asked about the possibility of using it, they foresee advantages on tasks such as: browsing photos and videos (75\%), adjust the tablet volume (78\%), play the media content (74\%), and perform speech-to-text tasks like writing emails (68\%) and notes $(71 \%)$. As a result, we will provide speech navigation for every interaction on the Tablet device. Moreover, seniors found interesting to interact with the tablet using touches on the back of the device. They recognized it could constitute an advantage when they need the screen to be completely visible (like playing/pausing a video (47\%)) and for basic tasks such as media content navigation (52\%). As a result, we considered exploring this interaction for tasks such as seeing extra information about a post. These results show that older adults are strongly interested in having alternative ways of interacting with a Tablet-based SNS, such as, voice, tilting and back-of-device touch. Additionally, they preserve the traditional interaction (touch and gestures) and are helped by contextual information in both audio, visual forms.

\subsection{Concerning the concepts of adaptation and personalization, which is preferred by seniors (RQ6)?}

Concepts of personalization and adaptation were explained to older adults before asking them about any preference. After acknowledging the concepts, they were asked about the possibility of changing content size, and increase/decrease audio volume within the application, both by doing it manually (personalization) or being done automatically (adaptation). Results show a strong interest in both features in both concepts (averaging from 4.10 to 4.35 for personalization, and from 4.29 to 4.32 for adaptation). Results obtained through a Likert scale from 1 (I think that is bad) to 5 (I think that is good) averaged $4.39(\mathrm{SD}=0.72, \mathrm{MED}=5.00$, mode $=5)$ for personalization and $4.32(\mathrm{SD}=1.013, \mathrm{MED}=5.00$, mode $=5)$ for adaptation. We also verified that there is not a clear preference for either one. As a result, we decided to implement personalization features related with increasing and decreasing interface elements and audio volumes, on both prototypes. These results indicate that seniors have a strong interest in adaptation and personalization concepts, showing preference for neither. 


\subsection{What issues should be taken into account in the implementation of adaptation and personalization concepts (RQ7)?}

We also observed older adults opinions about other conditions related with the concepts of adaptation and personalization. In general, they found interesting to have a technology tailored to their needs $(\mathrm{M}=3.52, \mathrm{SD}=1.48, \mathrm{MED}=4.00$, mode $=5)$ and would not mind if both adaptation (87\%) and personalization (83\%) would make their interfaces different from others. However, almost every participant (90\%) argue for the necessity of having control over what interface features could be adapted. Finally, when asked if they would mind if the technology collected data about the way they interact with it for adaptation purposes, participants showed tolerance $(M=3.90$, $\mathrm{SD}=1.30, \mathrm{MED}=4.00$, mode $=4$ ). Those with less tolerance justified it with concerns of privacy ("I do not know what "they" are going to do with my data"). These results indicate that older adults do not mind if adaptation and personalization make user interfaces different from others, although it is imperative that they retain control over what can be changed. Additionally, they do not mind if data about the way they interact with the systems is gathered for adaptation or personalization purposes.

\section{Developing TV and Tablet Interfaces}

In this section, we present both prototypes taking into account all the recommendations and results from the structured interviews highlighted in the previous section. Additionally, for a better understanding of what was validated or discussed on the focus groups, we will make a reference to that process every time we present a new feature.

\subsection{You, Me \& TV Prototype}

You, me \& TV prototype provides three different modalities (RC, voice and gestures) which were indicated during interviews with older adults. RC interaction is done with directional arrows and the OK button as previous research confirms that this is the best approach for older adults [7]. Additionally, remaining keys can be used to perform social tasks, such as making a post with a press of a button. Another modality implemented is speech recognition. This modality has two distinct approaches. The first is based on voice commands that perform a particular action depending on the current application context task. The second is based on speech-to-text. Both approaches were implemented using the Kinect microphone. The last modality is gesture recognition that allow users to perform gestures to control the application. We follow the mouse cursor metaphor approach, as Bobeth et al. [3] had confirmed through studies with senior users that this would be the best approach. We decided to validate two different ways to implement confirmation of actions with gestures. In the first option confirming is done through the hand closing. In the second option, conforming is done by leaving the "mouse cursor" three seconds over the object that user wants to select. Both approaches are being validated during the longitudinal tests. 


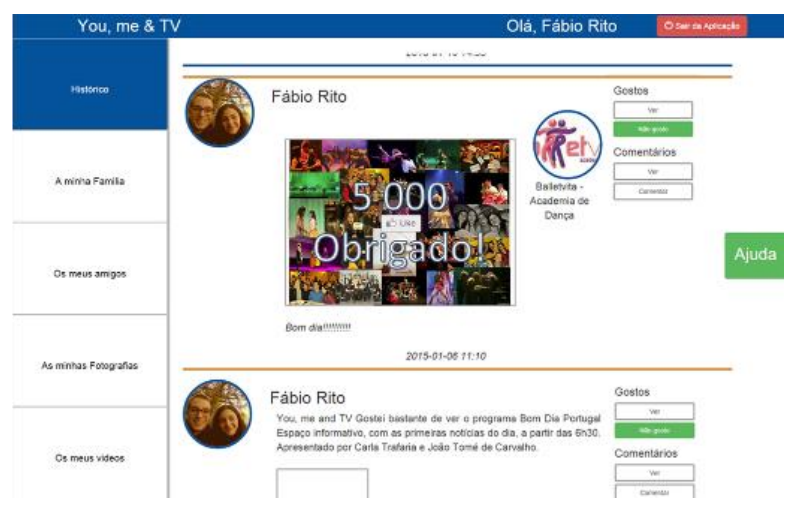

Fig. 5. TV-based prototype You, Me \& TV

The prototype (Figure 5) focuses on three main functionalities: content publishing, content visualization and group management. Content publishing functionality addresses the types of media content that is possible to share through TV technology. As it was possible to understand from interviews and focus groups, older adults enjoyed further to share photos and TV content with family and close friends. These two types of content lead seniors to mention two different usage contexts. The possibility to share printed photos that older users have in their albums; and the possibility to share what seniors are seeing on TV at a specific time. Both actions end with the posting of a publication on Facebook. Content visualization allows older users to see photos, videos and text content of their family and close friends. The prototype has two main areas, in one it is possible to see family members and in other to see friends, as requested in the focus groups. If a user wants to see information about a specific member he should select it and the prototype presents an area with all the information. The information is divided in photos, videos, publications and general profile and interests. This division was also informed by older adults in the focus groups. Finally, the third main functionality allows users to manage sub groups inside family and close friends' screens. This was a surprising feature raised by older adults as they wanted to create sub groups inside family and friends. They gave us some examples like "I had gymnastics friends and coffee friends and I want to distinguish these two groups". We also perceived that if a friend belongs to two groups then it should appear in both groups.

\subsection{YouTablet Prototype}

The YouTablet prototype (Figure 5 (right)) offers five modalities of interaction: touch, gesture, speech, tilting and back-of-device touch. As requested on the focus groups, touch interaction will be based on providing touch buttons for every possible action. Additionally, gestures will be supported as intuitive options to accomplish the same actions. Speech interaction, like on the You, Me \& TV prototype, will be based on both voice commands and speech-to-text functionalities using the Android speech recognition module. Tilting will be offered as an exploratory interaction, based on a 
"press to tilt" concept where the user has to press a button on the interface and at the same time tilt the device. This concept was inspired by both the "squeeze and tilt" concept [18], and Rekimoto concept for tilting operations on small screen devices [25]. Tilting will be offered for menu navigation and scrolling tasks, as suggested by older adults opinions on both the interviews and the focus groups - they perceived it as very useful and easy to do, however they expressed the need for help on the first interaction, especially regarding what button to press to begin the interaction -. Finally, and also as a result of the structured interviews, YouTablet will support back-ofdevice interaction inspired by Robinson et al. [26] for tasks related with getting extra information from interface items. For implementing this feature we followed an approach based on a state machine algorithm (Figure 6).

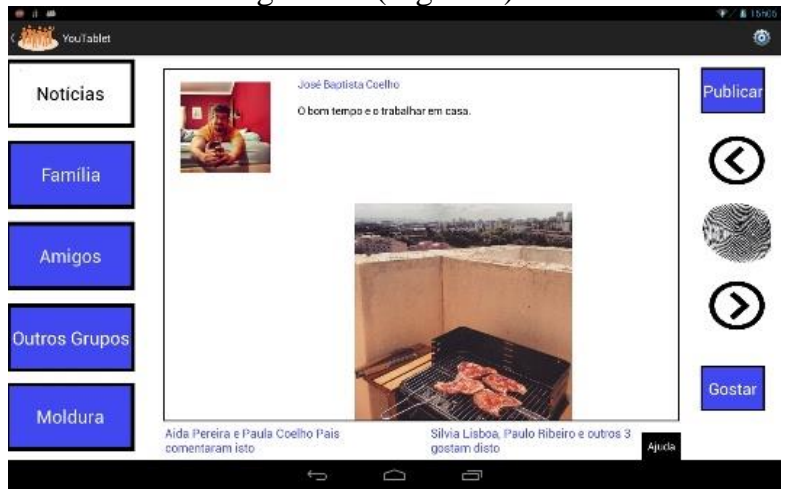

Fig. 6. Tablet-based Facebook prototype (right).

Regarding functionalities, YouTablet focuses on content publishing, content visualization, group visualization and digital photo frame. Content publishing is based on providing the traditional posting options already available in Facebook; YouTablet approach on content visualization is based on presenting posts one by one. Each post's content is fitted to the screen in order to focus older adults' attention. This concept was validated on the focus groups, being approved by all participants. Navigation through posts is based on a next and previous approach, and supported by four distinct modalities of interaction: gestures, voice commands, buttons by touch and tilting. In the focus groups, swipe was seen as the main modality to achieve this navigation. It was also referred the need for providing help throughout the application regarding which voice commands could be used. Button navigation also seemed intuitive, although some participants expressed the desire for having buttons on each side of the content, even at the expense of having to grab the tablet only with one hand. As a result, the location of the buttons could be considered as a personalization option. Still, regarding content visualization, flipping the post to see its comments and likes was implemented as it provides more space to organize the graphic elements on interface and allow for a larger font. Back-of-device touch will be offered to achieve this, as focus groups participants perceived it as a very natural action. Additionally, this can also be achieved by using buttons labelled "See comments" and "See likes". Regarding group visualization, YouTablet will offer the possibility of filtering posts by 
groups and navigating through its members. Additionally, options for managing groups suggested in the You, Me \& TV prototype were also validated by the participants. Digital photo frame is a slide show mode, which presents the Family group members photos like a digital frame. It has an automatic activation and deactivation. When the tablet is not being used, it starts the slide show until the user grabs the tablet again. This functionality was first perceived as a good idea on the interviews and further validated on the focus groups. The latter participants requested an option to directly access the content being shown, and an option for also visualizing events. Finally, concerning the top action bar and the existence of symbols as shortcuts for features, participants pointed out the need for additional help in understanding its purposes or the addition of text labels.

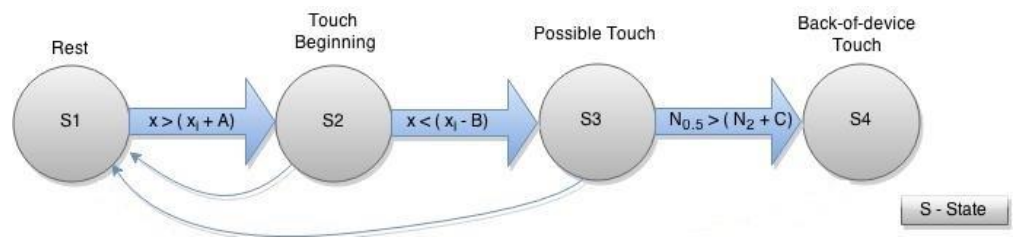

Fig. 4. The back-of-device touch state machine: from a rest position (S1), the algorithm measures the device spatial variation on the 3 axis. When a positive peek A first happens, the machine transits to S2. Then, when a peek B happens in the opposite direction, the machine reaches S3. Finally, if the device's microphone detects a sound variation of at least $\mathrm{C}$ it reaches S4. Reaching S4 means the machine recognized a touch. In S2 and S3, if the transition condition is not met in a set amount of time, the machine goes back to $\mathrm{S} 1$

\section{Conclusion}

Social isolation seriously damages the health of older adults leading to a progressive increase in the risk of mortality. Facebook has the potential to increase the number of social interactions of older people with relatives and friends by getting them to feel less isolated. We conducted a study consisting of 30 semi-structured interviews and 3 focus groups. In the interviews we sought to differentiate older adults who use Facebook from the ones who do not, and to perceive the opportunities for using multimodal adaptive TV and tablet devices as ways for non-users of SNS to gain access to them. Focus groups allowed to involve older people in the design of Facebook prototypes for TV and tablet. The results showed that the elderly are receptive to using SNSs on TV and Tablet and that these applications must meet a number of recommendations: they should provide different modalities like RC, voice and gestures for TV, and touch, gestures and voice for Tablet; exploratory modalities like tilting and back-of-device touch can also be used for menu navigation and showing contextual information on Tablet; older adults highlighted the importance of always getting contextual help through several outputs and appreciate the concepts of adaptation and personalization. Finally, the study also suggested new features such as sharing of printed photos through the TV-based prototype and a digital photo frame through a Tablet-based interface which presents the family members photos in a slide show 
mode like a digital frame. Both prototypes and design decisions are being further validated through a longitudinal study.

\section{Acknowledgments}

We would like to thank to Universidade Sénior de Massamá e Monte Abraão and all the participants for making this study possible. This work was partially supported by Fundação para a Ciência e Tecnologia (FCT) through Multiannual Funding to the LaSIGE research unit and the Individual Doctoral Grant SFRH/BD/81115/2011.

\section{References}

1. Top 15 Most Popular Social Networking Sites - January 2015 (2015), http://www. ebizmba.com/articles/social-networking-websites

2. Alaoui, M., Lewkowicz, M.: A livinglab approach to involve elderly in the design of smart TV applications offering communication services. Lecture Notes in Computer Science, vol. 8029, pp. 325-334. Springer Berlin Heidelberg (2013)

3. Bobeth, J., Schmehl, S., Kruijff, E., Deutsch, S., Tscheligi, M.: Evaluating performance and acceptance of older adults using freehand gestures for TV menu control. pp. 35-44. EuroiTV '12, ACM, New York, NY, USA (2012)

4. Bobeth, J., Schrammel, J., Deutsch, S., Klein, M., Drobics, M., Hochleitner, C., Tscheligi, M.: Tablet, gestures, remote control?: Influence of age on performance and user experience with iTV applications. pp. 139-146. TVX '14 (2014)

5. Burke, M., Kraut, R., Marlow, C.: Social capital on facebook: differentiating uses and users. pp. 571-580. CHI'11 (2011)

6. Chen, Y.: Usability analysis on online social networks for the elderly. Tech. rep., Helsinki University of Technology (2009)

7. Coelho, J., Biswas, P., Duarte, C., Guerreiro, T., Langdon, P., Feiteira, P., Costa, D., Costa, D., Neves, B., Alves, F.: Involving all stakeholders in the development of TV applications for elderly. International Journal On Advances in Intelligent Systems 5(3 and 4), 427-440 (2012)

8. Coelho, J., Duarte, C., Biswas, P., Langdon, P.: Developing accessible TV applications. pp. 131-138. ASSETS '11, ACM (2011)

9. Cornejo, R., Tentori, M., Favela, J.: Enriching in-person encounters through social media: A study on family connectedness for the elderly. Int. J. Hum.-Comput. Stud. 71(9), 889-899 (Sep 2013)

10. Cornwell, E.Y., Waite, L.J.: Measuring social isolation among older adults using multiple indicators from the nshap study. Journals of Gerontology: 64B(suppl 1), i38-i46 (2009)

11. Findlater, L., Wobbrock, J.: Personalized input: improving ten-finger touchscreen typing through automatic adaptation. In: Proc. CHI 2012. pp. 815-824. ACM (2012)

12. Gajos, K.Z., Wobbrock, J.O., Weld, D.S.: Automatically generating user interfaces adapted to users' motor and vision capabilities. pp. 231-240. UIST '07 (2007)

13. Garattini, C., Wherton, J., Prendergast, D.: Linking the lonely: An exploration of a communication technology designed to support social interaction among older adults. Univers.Access Inf. Soc. 11(2), 211-222 (Jun 2012)

14. Gaver,W., Boucher, A., Bowers, J., Blythe, M., Jarvis, N., Cameron, D., Kerridge, T.,Wilkie, A., Phillips, R., Wright, P.: The photostroller: Supporting diverse care home residents in engaging with the world. pp. 1757-1766. CHI '11, ACM (2011) 
15. Gibson, L., Moncur, W., Forbes, P., Arnott, J., Martin, C., Bhachu, A.S.: Designing social networking sites for older adults. pp. 186-194. BCS '10, British Computer Society, UK.

16. Gomes, G., Duarte, C., Coelho, J., Matos, E.: Designing a facebook interface for senior users. The Scientific World Journal Article ID 741567, 8 pages (2014)

17. Grosinger, J., Vetere, F., Fitzpatrick, G.: Agile life: Addressing knowledge and social motivations for active aging. pp. 162-165. OzCHI '12 (2012)

18. Harrison, B.L., Fishkin, K.P., Gujar, A., Mochon, C., Want, R.: Squeeze me, hold me, tilt me! an exploration of manipulative user interfaces. pp. 17-24. CHI '98 (1998)

19. Judge, T.K., Neustaedter, C., Harrison, S., Blose, A.: Family portals: Connecting families through a multifamily media space. pp. 1205-1214. CHI '11 (2011)

20. Karahasanovi, A., Brandtzg, P.B., Heim, J., Lders, M., Vermeir, L., Pierson, J., Lievens, B., Vanattenhoven, J., Jans, G.: Co-creation and user-generated contentelderly peoples user requirements. Computers in Human Behavior 25(3), 655 - 678 (2009)

21. Lindley, S.E., Harper, R., Sellen, A.: Desiring to be in touch in a changing communications landscape: attitudes of older adults. In: Proc. CHI 2009. pp. 1693-1702

22. Lindley, S.E.: Shades of lightweight: Supporting cross-generational communication through home messaging. Univers. Access Inf. Soc. 11(1), 31-43 (Mar 2012)

23. McLaughlin, A.C., Rogers, W.A., Fisk, A.D.: Using direct and indirect input devices: Attention demands and age-related differences. ACM Trans. Comput.-Hum. Interact. 16(1), 2:12:15 (2009)

24. Norval, C., Arnott, J.L., Hanson, V.L.: What's on your mind?: Investigating recommendations for inclusive social networking and older adults. pp. 3923-3932. CHI '14, ACM, NY

25. Rekimoto, J.: Tilting operations for small screen interfaces. pp. 167-168. UIST '96 (1996)

26. Robinson, S., Rajput, N., Jones, M., Jain, A., Sahay, S., Nanavati, A.: Tapback: Towards richer mobile interfaces in impoverished contexts. pp. 2733-2736. CHI '11 (2011)

27. Stephanidis, C., Paramythis, A., Sfyrakis, M., Stergiou, A., Maou, N., Leventis, A., Paparoulis, G., Karagiannidis, C.: Adaptable and adaptive user interfaces for disabled users in the avanti project. pp. 153-166. IS\&N' '98 (1998)

28. Stobel, C., Blessing, L.: Mobile device interaction gestures for older users. pp. 793-796. NordiCHI '10 (2010)

29. Waycott, J., Vetere, F., Pedell, S., Kulik, L., Ozanne, E., Gruner, A., Downs, J.: Older adults as digital content producers. In: Proc. CHI 2013. pp. 39-48. ACM (2013) 